\title{
Differential immune responses of a novel Toll gene to Vibrio parahaemolyticus, LPS and Poly I:C in noble scallop Chlamys nobilis
} with different carotenoid contents

\author{
Huaiping Zheng ${ }^{\mathrm{a}, b^{*}}$, Yeqing $\mathrm{Lu}^{\mathrm{a}, \mathrm{b}}$ \\ a Key Laboratory of Marine Biotechnology of Guangdong Province, Shantou \\ University, Shantou 515063, China \\ ${ }^{\mathrm{b}}$ Mariculture Research Center for Subtropical Shellfish \& Algae, Shantou University, \\ Shantou 515063, China
}

\begin{abstract}
The noble scallop Chlamys nobilis, an important farming shellfish in the southern sea of China since 1980s, has suffered large-scale mortality problems in recent years. As efficient antioxidants, carotenoids have been reported to protect cells and tissues from oxidative damage due to adversity stress by eliminating the active oxygen free radicals. To investigate whether carotenoids have an immune influence to $C$. nobilis under pathogen stress, two stain scallops of orange and brown with different carotenoids content were used in this study. Carotenoids content in scallops' muscle of the orange strain is significantly higher than that of the brown strain. The two strain scallops had the same age and been cultured the same environmental conditions. 300 scallops including 150 orange and 150 brown were firstly chosen from the field, then randomly divided into eight $500 \mathrm{~L}$ tanks (36 scallops each tank) for aerating culture. After $24 \mathrm{~h}$ acclimatization, scallops suffered from an acute challenge lasting $48 \mathrm{~h}$ of Vibrio parahaemolyticus, lipopolysaccharide (LPS), polyinosinic polycytidylic acid (Poly I:C), and PBS, respectively. Last, transcripts of a toll-like receptor called CnTLR-1, total carotenoids content (TCC), SOD activity, and CAT activity were measured. The full length cDNA of CnTLR-1 was 2982 bp with an open reading frame (ORF) of $1920 \mathrm{bp}$ encoding 639 deduced amino acids, which contained five leucine-rich repeats (LRR), two LRR-C-terminal (LRRCT) motifs, a LRR-N-terminal (LRRNT) motif in the extracellular domain, a transmembrane domain and a Toll/Interleukin-1 Receptor (TIR) of 138 amino acids in the cytoplasmic region. CnTLR-1 was closely related to Crassostrea gigas and Mytilus galloprovincialis Tolls. CnTLR-1 transcripts from higher to lower in turn expressed in mantle, haemocytes, gill, kidney, gonad, hepatopancreas, intestines and adductor. Compared with PBS challenge, CnTLR-1 transcripts were all up-regulated by $V$. parahaemolyticus, LPS and Poly I:C, and transcripts were significantly higher in orange scallops than that of brown ones in all the immune challenges. TCC from the orange scallops were significantly higher than that from the brown ones in all tested tissues and first increased and then decreased. SOD enzymatic activities showed V-shapes and were lower in orange scallops than in brown ones, while CAT enzymatic activities
\end{abstract}


displayed inverted V-shapes and were higher in orange scallops than in brown ones. The decrease of SOD and the increase of CAT were significantly higher in orange scallops than brown ones. The present results indicate that CnTLR-1 is an important factor involved in the immune defense against pathogens, and carotenoids can play roles in antioxidant defense system by up-regulating gene expression under pathogen stress in the noble scallop.

Keywords: The noble scallop Chlamys nobilis; Toll-like receptor; carotenoids; Vibrio parahaemolyticus.

Funding for this research was provided by National Natural Science Foundation of China (31372528), China Modern Agro-industry Technology Research System (CARS-48), Guangdong Province Natural Sciences Foundation (2015A030311027), Science \& Technology Plan (2013B020503061, 2015B090903081) and Marine and Fishery Promotion Project (B201300B06), China.

${ }^{*}$ Corresponding author: Tel: 0086-754-86503285 Fax: 0086-754-86500614

E-mail address: hpzheng@stu.edu.cn 